\section{Tuberkulose: Massen-Screening nicht sinnvoll}

Herzmann C et al. Radiological screening of refugees in Germany. Eur Resp J 2017; 49: 1602487. doi:10.1183/13993003.02487-2016

2015 kamen mehr als 1 Million Flüchtlinge in Deutschland an. Gleichzeitig nahmen Tuberkulose-Diagnosen bei Migranten zu. Das Asylverfahren- und Infektionsschutzgesetz sehen bei einer Unterbringung in Gemeinschaftsunterkünften und Aufnahmeeinrichtungen das Röntgen des Thorax vor, um eine Tuberkulose frühzeitig zu erkennen. Herzmann et al. bezweifeln die Effektivität der generellen Reihenuntersuchung für Asylbewerber.

Der Arbeitsgruppe standen Röntgenbefunde von 38001 Asylsuchenden aus 4 deutschen Erstaufnahmeeinrichtungen zur Verfügung. Die größte Aufgabe bewältigte Karlsruhe mit 30228 Ankömmlingen. Das Durchschnittsalter der Menschen betrug 27, 1 Jahre und 25,8\% wa-

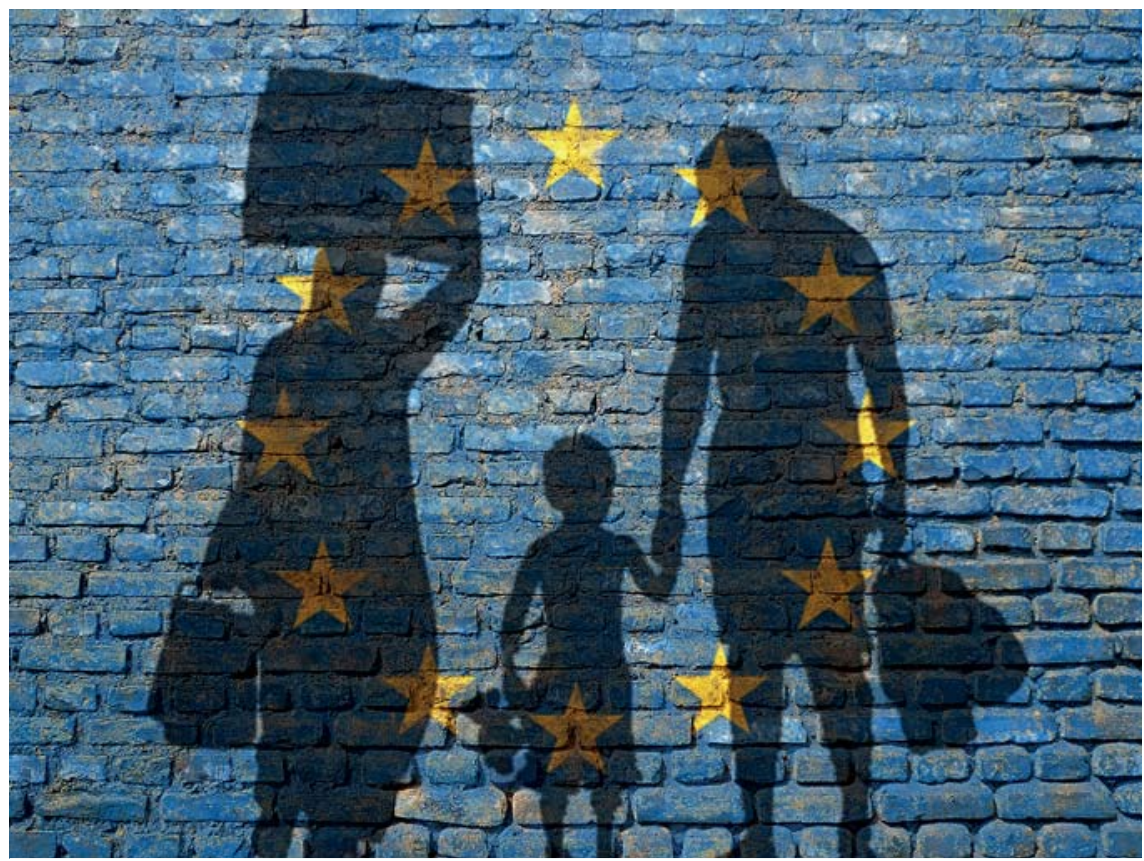

- Das Screening auf infektiöse Tuberkulose dient in erster Linie dem Schutz der Asylsuchenden selbst sowie enger Kontaktpersonen und erlaubt eine frühzeitige Einleitung der Therapie von identifizierten Erkrankten. Bildquelle: Jonathan Stutz/www.Fotolia.com ren weiblich. Die häufigsten von insgesamt 76 Herkunftsländern waren Syrien (25\%), Irak (14\%) und Afghanistan (11\%). 127 Röntgenberichte ergaben den Verdacht auf eine Tuberkulose. 37 Betroffene gingen der Nachbeobachtung verloren. In 38 Fällen schlossen weiterführende Untersuchungen eine Tuberkulose aus. Bei 52 Personen bestätigte sich die Erkrankung und die Einleitung einer Therapie folgte. Die mikrobiologische Bestätigungsrate betrug $81 \%$ und war mit den niederländischen Erfahrungen vergleichbar. Die Gesamtprävalenz lag bei 140/100000 Menschen. Für die einzelnen Bevölkerungsgruppen stieg die Prävalenz mit den Krankheitsraten im Heimatland. Dementsprechend führten ostafrikanische Länder die Liste an. Die Inzidenz bei den Personen aus Somalia und Eritrea übertraf die Schätzungen der WHO um das 4- bis10-Fache.

- Somalia 1075/100000,

- Eritrea 640/100000,

- Gambia 336/100000,

- Pakistan 234/100000,

- Osteuropa 166/100000,

- Afghanistan 139/100000,

- Irak 37/100000,

- Syrien 31/100000.
Um den Nutzen des Massen-Screenings in Deutschland zu analysieren, stellten die Autoren eine repräsentative Gruppe von Migranten zusammen. Die deutsche Einwanderungsstatistik ergab ein ähnliches Länderbild wie die Studie. Die meisten Flüchtlinge kamen aus

- Syrien $37 \%$,

- Irak 14\%,

- Afghanistan 11\%,

- Pakistan $2 \%$,

- Eritrea $2 \%$.

Die Zahl der benötigten Röntgenbilder, um 1 Tuberkulose zu diagnostizieren, betrug für Syrer 3000 und für Somalier 94 Thoraxaufnahmen. Die Relevanz einer Tuberkuloseübertragung durch unerkannte Fälle sei unklar. Das Screening modifiziere wahrscheinlich stärker die individuelle Krankheitsentwicklung und -sterblichkeit als die Epidemiologie.

\section{FAZIT}

Die Autoren meinen: 1. Bei der Indikation zu einem Röntgenbild sollte das Herkunftsland des Asylsuchenden Berücksichtigung finden. Bei vergleichsweise wenigen afrikanischen Flüchtlingen war die Thoraxdarstellung effizienter als bei vielen Schutzsuchenden aus Vorderasien. 2. Die hohe Ausfallquote im Follow-Up könne zu einer Unterschätzung der Tuberkuloseinzidenz und Datenverzerrung geführt haben. Eine kontinuierliche Beobachtung in der hochmobilen Risikopopulation sei schwierig, aber essenziell.

Dr. med. Susanne Krome, Melle 